\title{
An alternative view of enzyme catalysis ${ }^{\star \star \star \star}$
}

\author{
Fredric M. Menger \\ Department of Chemistry, Emory University, Atlanta, GA 30322, USA
}

\begin{abstract}
This paper begins with a brief review of theories and concepts that have influenced today's view of enzyme catalysis: transition-state stabilization, entropy, orbital steering, proximity, and intramolecularity. The discussion then launches into the "spatiotemporal" model of enzyme catalysis in which fast intramolecular and enzymatic rates are ascribed to short distances that are imposed rigidly upon the reacting entities. An equation relating rate and distance is set forth, as are experimental and computational data supporting this relationship. Finally, enzyme systems themselves are analyzed in terms of the distance parameter and the so-called "split-site" model in which ground-state geometries play a crucial role. Among the many surprising conclusions is a transition-state stabilization by noncovalent forces (e.g., hydrogen-bonding) that are positioned far away from the actual transition-state chemistry. The model also confronts and dismisses the claim in classical enzymology that the ubiquitous enzyme-substrate complex is either inconsequential or inhibitory to the overall reaction rate.
\end{abstract}

Keywords: Enzyme catalysis; transition-state stabilization; orbital steering; spatiotemporal model; split-site model.

I have a friend who has a little dog that likes to mark trees. It usually bypasses trees that have not been already marked by other canines, but when it encounters a highly frequented tree, it does its best to impart its own essence. In fact, the dog tries hard to mark higher up on the tree than have other dogs previously. Since my friend's dog is small, the effort to reach high often causes the animal to tumble, rather comically, over on its back. Science is a bit like that. There is unmarked science and marked science. Unmarked science has the advantage of having fewer competitors, and the possibilities of new insights are favorable. Marked science receives more attention and funding, but there is always the risk of "tumbling" in the presence of a "taller dog". Enzyme catalysis, with its countless worldwide publications, is definitely a marked science. Philosophically, we have favored unmarked science (having been heavily engaged in colloidal organic chemistry, a comparatively lonely enterprise). But, from time to time, we have visited enzymes [1-10], and the ensuing paper describes the results. Readers can judge for themselves how high up we have marked.

\section{TRANSITION-STATE STABILIZATION}

The story begins with a 1948 Nature paper by Linus Pauling [11] in which he writes: "I think that enzymes are molecules that are complementary in structure to the activated complexes of the reactions that they catalyze." This statement expresses the idea, still generally accepted today, that enzyme reac-

\footnotetext{
*Paper based on a presentation at the $17^{\text {th }}$ International Conference on Physical Organic Chemistry (ICPOC-17), Shanghai, China, 15-20 August 2004. Other presentations are published in this issue, pp. 1807-1921.

**This work was supported by the National Institutes of Health.
} 
tions are fast because the transition state is stabilized. By "fast" is meant $10^{8}-10^{12}$ times faster than in the absence of an enzyme. The energy diagram in Fig. 1 illustrates the point. Thus, as indicated by the arrow, an enzyme manages to lower the transition-state energy from that of an uncatalyzed reaction to that found within the enzyme-substrate complex ES.

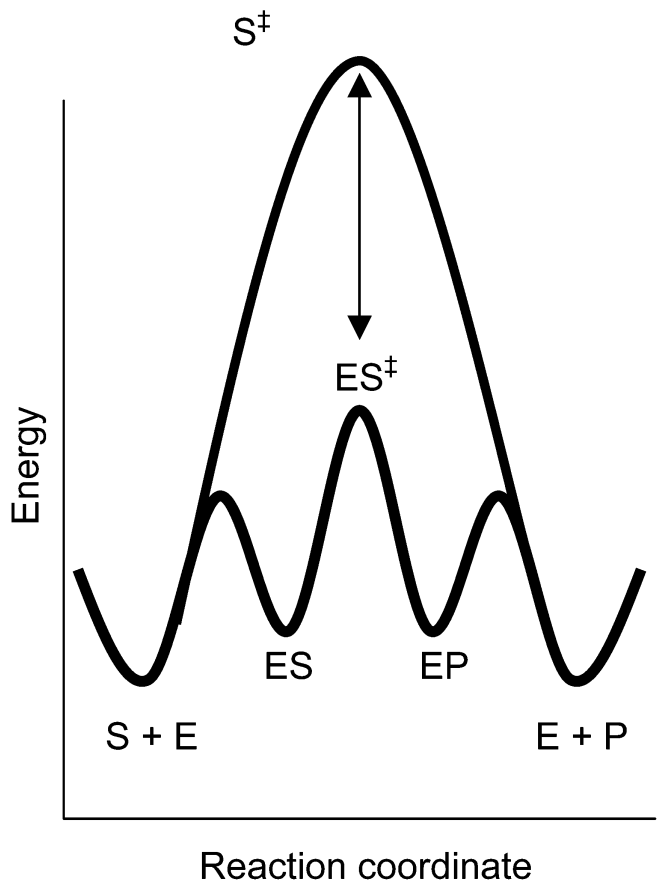

Fig. 1 Energy profiles showing a lowering of the transition-state energy from an uncatalyzed reaction (upper curve) to enzyme-catalyzed (lower curve) reaction.

One of the best available examples of an enzyme's transition-state stabilization comes from the proteases that hydrolyze peptides (Fig. 2). In this hydrolysis, the amide's carbonyl oxygen is seen to become fully anionic in the tetradhedral intermediate. This implies that the oxygen assumes a partially anionic charge in the transition state leading to the intermediate. The developing anionic charge is then

\section{Oxyanion hole}
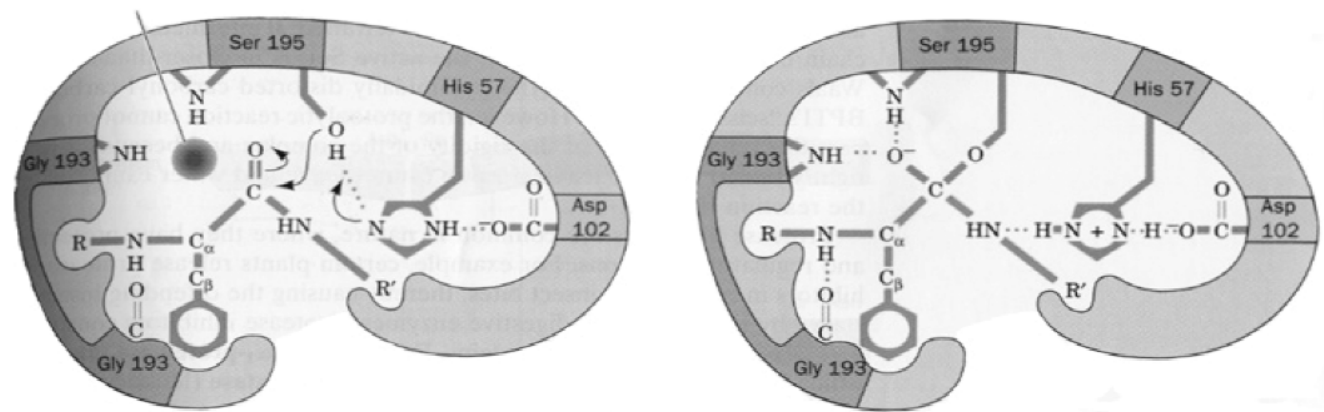

Fig. 2 Transition-state stabilization of a developing anionic charge on a carbonyl oxygen within the oxyanion hole of a protease. Reproduced with permission from D. Voet, J. G. Voet, and C. W. Pratt, Fundamentals of Biochemistry. 
stabilized by hydrogen-bonding in the so-called "oxyanion hole" at the active site. What is less clear is the magnitude of (a) the partial negative charge on the carbonyl oxygen at the transition state and (b) the rate reduction resulting from the hydrogen-bonding within the oxyanion hole. Yet, qualitatively at least, it is clear that Fig. 2 represents a good illustration of the Pauling notion.

Houk et al. [12] state that "transition states must be bound better than substrates...for catalysis to be effective." Briefly, if the enzyme-substrate association constant is $10^{4} \mathrm{M}^{-1}$, then they calculate that the enzyme-transition-state binding must be $10^{16} \mathrm{M}^{-1}$ in order to explain a $10^{12}$-fold catalysis. The $10^{16} \mathrm{M}^{-1}$ binding constant for the fleeting transition state is astounding. But just as astounding is the fact that the enzyme ostensibly discriminates between the ground state and the transition state (which often resemble each other closely) by a factor of $10^{12}$. It is difficult, however, to escape these huge numbers if one accepts transition-state stabilization as the sole source of catalysis.

I will return to the matter of transition-state stabilization toward the end of this paper, but for the moment let me point out that transition-state stabilization is not the only way a reaction can be accelerated. One can also destabilize the ground state. It is, of course, not a matter of deciding whether a catalysis originates from transition-state stabilization or, alternatively, from ground-state destabilization. Both may play a role in enzymology.

\section{ENTROPY}

Entropy is a popular rationalization for fast enzymatic reactions, witness the quote from Biochemistry by Abeles, Frey, and Jencks [13]: "We can conclude that one effect of substrate binding at the active site of an enzyme is to increase the probability for the reaction and decrease the requirement for loss of entropy, and this can account for large rate increases in the reaction of substrates." The idea here is that bringing two reactive entities together prior to reaction normally causes a loss of entropy that impairs the reaction rate. When, however, a substrate is held tightly at an active site of an enzyme, there is no longer a need to freeze translational and rotational motions, and thus the ensuing reaction between enzyme and substrate is accelerated. Enzyme catalysis is, according to this school of thought, all in the entropy.

In my view, the entropy theory is both correct and useless. Validity and utility in science are not necessarily interrelated! Table 1 illustrates the point. In the anhydride formation, the only difference among the reactions is the number and type of alkyl groups on the compounds. Yet, the entropies of activation $\Delta \mathrm{S}^{*}$ range inexplicably from +2.9 to -8 eu. Since the magnitudes, and even the signs, of the entropies are unpredictable, entropies are not generally useful in rationalizing rates of reactions in solution. This is seen also in the two $\mathrm{S}_{\mathrm{N}} 2$ reactions in which alkoxides displace cationic sulfurs. Similar though the reactions might be, one $\Delta \mathrm{S}^{*}$ equals $-3.7 \mathrm{eu}$, while the other $\Delta \mathrm{S}^{*}$ equals $+14 \mathrm{eu}$.

Why should solution reactions have activation entropies that are so erratic? The reason is that entropy, unlike time and distance parameters, is a hodge-podge of multiple effects including motional freedom and (especially troublesome) solvation. Each reaction has its own particular, and unfathomable, combination of entropy components. Bird and Stirling [14] expressed it thusly: "Activation parameters...do not accord with any simple ideas of the factors which control rates of cyclization." And DeTar and Luthra [15] wrote, "There is no simple way to summarize idiosyncratic contributions of individual structures to the enthalpies and entropies of activation." Should there still be any doubt as to entropy's lack of utility when applied to enzymes, one need only attempt to define the entropy of an active site.

Claude Shannon, a famous developer of information theory, invented an uncertainty function in communications engineering. Needing a name for his function, he went to John Von Neumann, a renowned mathematician, for advice. 
Table 1 Activation entropies for two reactions.

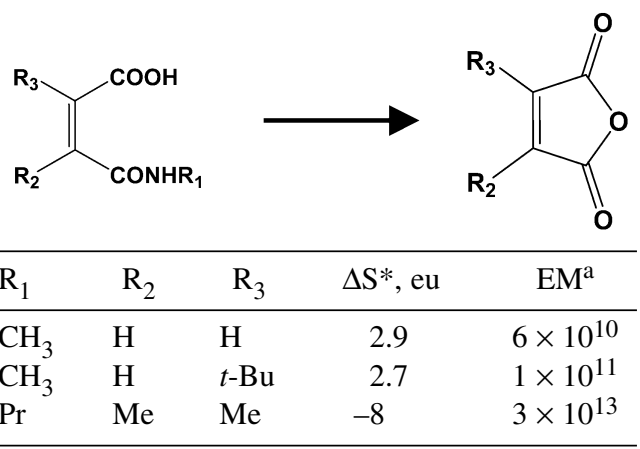

${ }^{\mathrm{a}} \mathrm{EM}=k_{\text {intra }} / k_{\text {inter }}$
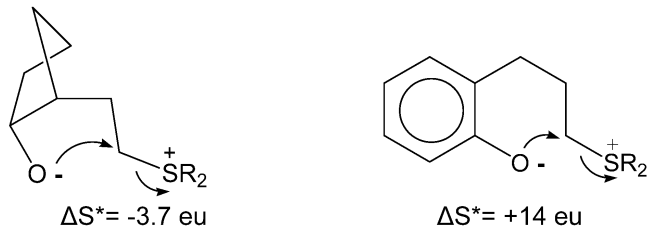

Von Neumann told him, "Call your new function entropy. Since no one really knows what entropy is, in a debate you will always have an advantage." Another illustrative quote comes from our own writings: "Saying that an enzyme reaction is fast because of entropy is like saying that a day is hot because of climate. Both statements are true, but both contain little information." In summary, the assertion that "enzymatic catalyses are entropic in origin," while not directly incorrect, does little to help our understanding of enzyme mechanism.

\section{ORBITAL STEERING}

Orbital steering, proposed by Storm and Koshland in 1972 [16], was a clever explanation of enzyme catalysis. The idea is that a successful reaction occurs only after overlap of the "reactive cones" of the two relevant orbitals. These reactive cones are represented by the dark wedges in Fig. 3. Simple geometric considerations show that if the cones' solid angles are only $10^{\circ}$, then bringing the two cones into contact results in a $10^{4}$-fold rate increase (relative to when the reactive surfaces cover the entire spheres, and thus all random collisions are productive). If the reactive cones are indeed small, and if two such pairs of overlapped cones are present at the active site, then a $10^{8}$ catalysis, typical of many enzymes, can be achieved.

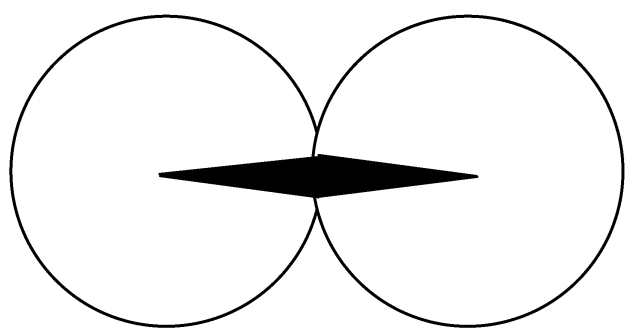

Fig. 3 Orbital steering in which reactive cones (black wedges) of two orbitals are forced to overlap. 
Orbital steering caused an immediate negative outcry. It was claimed, on theoretical grounds, that reactive cones are much larger than $10^{\circ}$ and that, therefore, the benefit of enforced orbital proximity is much less than $10^{4}$. While not disagreeing with this assertion, we were puzzled that no one had ever bothered to test the point experimentally. We therefore set out to do so (in what still remains as one of my favorite personal experiments [17]). Figure 4 shows two hydroxyacids whose lactonization rates were determined. (Actually, additional compounds were investigated, but the two compounds in Fig. 4 serve to make the point). The hydroxyacids were ideal for testing the angle dependency of an organic reaction because the nucleophile/electrophile distances (i.e., the $\mathrm{O}_{1} / \mathrm{C}_{2}$ distances) are identical as are the strains in the two lactone products. On the other hand, the "attack angles" $\left(\mathrm{O}_{1} / \mathrm{C}_{2} / \mathrm{C}_{3}\right)$ differ by $10^{\circ}$. If Storm and Koshland are correct, then the rates of lactonization should differ by many orders of magnitude. But, to the contrary, the hydroxyacids lactonize at the same rate. The angle dependence clearly encompasses reactive cones that are much larger than $10^{\circ}$, thereby invalidating, at least for carbonyl additions, the orbital steering concept.

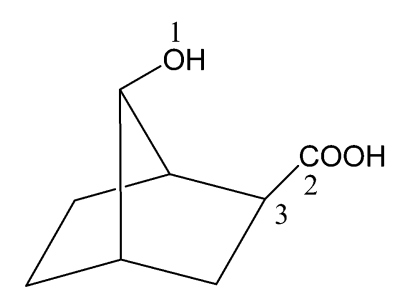

$$
\begin{aligned}
& \text { Angle }=70^{\circ} \\
& \mathrm{k}_{\text {rel }}=1.0
\end{aligned}
$$

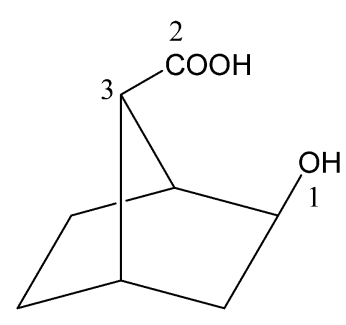

Angle $=80^{\circ}$

$\mathrm{k}_{\mathrm{rel}}=1.2$

Fig. 4 Two hydroxyacids used to test the orbital steering concept.

\section{PROXIMITY}

Bruice called "proximity" the common sense parameter [18]. We agree.

It is intuitively reasonable that both intramolecular and enzymatic reactions are fast because the reactive entities are held close to each other. There are, however, two problems with the proximity concept. First, in almost no case has proximity ever been defined quantitatively. Are two atoms in "proximity" when they are $0.25 \mathrm{~nm}$ apart? Or $0.50 \mathrm{~nm}$ ? Or $2.5 \mathrm{~nm}$ ? In other words, the proximity concept seems substantive but poorly defined.

A second problem with proximity was demonstrated by us [2] in a simple experiment in which methyl iodide was reacted with pyridine (eq. 1). The $\mathrm{S}_{\mathrm{N}} 2$

$$
\mathrm{CH}_{3} \mathrm{I}+\mathrm{Pyr} \rightarrow \mathrm{Pyr}^{+}-\mathrm{CH}_{3}+\mathrm{I}^{-}
$$

reaction was run with a low concentration of methyl iodide in a large excess of pyridine co-mixed with dichlorobenzene (a solvent with a polarity very similar to that of pyridine). As the pyridine concentration in the dichlorobenzene was increased, the rate of $k_{\text {obs }}$ increased linearly with the pyridine, as expected. The important point is that the linearity persisted up to $100 \%$ pyridine. In other words, there was nothing special about the reaction rate even when the methyl iodide substrate was "bathed" in nothing but pyridine. Total proximity between the reactants was assured, yet it was not associated with a special rate effect. I conclude that proximity (whatever that term means) is necessary, but not sufficient for an enzyme-like rate. The nature of this insufficiency will become clear later on in the paper. 


\section{INTRAMOLECULARITY}

Since intramolecular reactions can be much faster than their intermolecular counterparts, it was quite natural to relate enzymatic reactions to intramolecularity. Thus, intramolecular reactions are fast because covalent bonds hold the reactive entities in proximity (there's that word again!), while enzymatic reactions are fast because noncovalent forces in the ES complex do the same thing. The connection between enzyme catalysis and intramolecularity is no doubt valid, yet once again I must inquire as to the information content. One problem is that the acceleration associated with intramolecular reactions can vary by nine orders of magnitude (Table 2). Until we better understand the source of these huge variations, intramolecularity will not be as helpful in explaining enzyme action as one might hope.

Table 2 Variations in effective molarities for several intramolecular reactions ${ }^{\mathrm{a}}$.

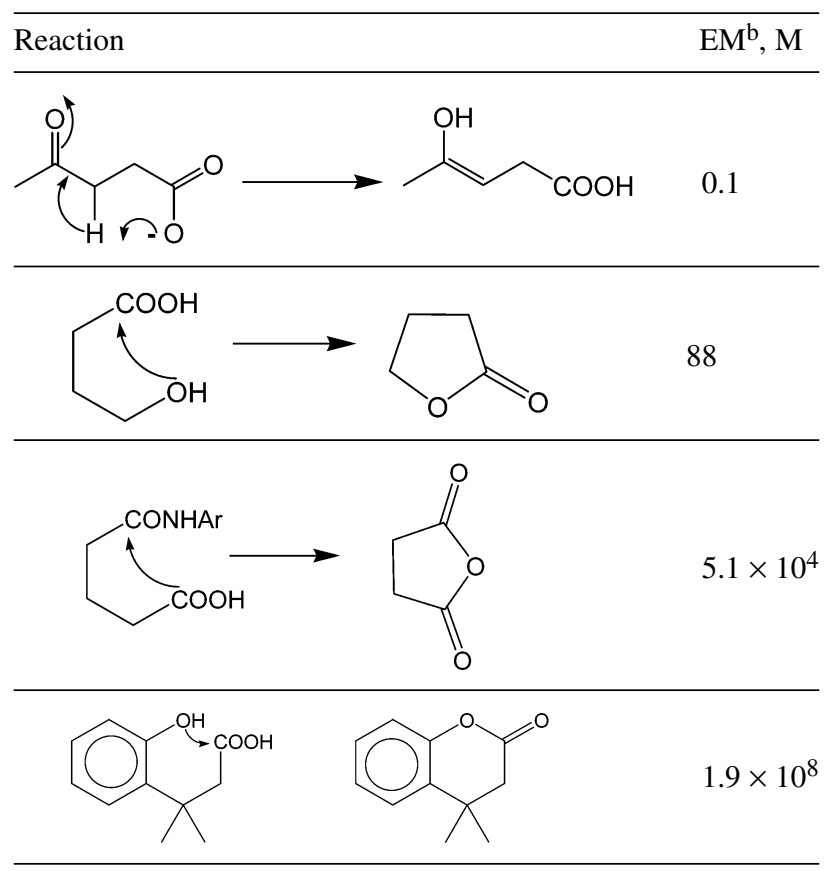

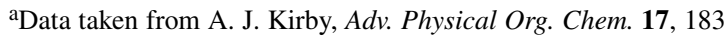
(1980).

bEM = "effective molarity" $=k_{\text {intra }} / k_{\text {inter }}$.

\section{SPATIOTEMPORAL EFFECTS}

In surveying long lists of intramolecular reactions for hints as to why they can achieve, in certain cases, enzyme-like accelerations, we observed an important principle: Sustained proximity at close distances leads to very fast rates and unique chemistry [1-10]. Thus, time and distance (to be defined more specifically later) are the key parameters. Two examples are shown in Fig. 5 [19]. In the first example, an amine attacks an electron-rich double bond to close a ring. Now textbooks teach that double bonds react with electrophiles, not nucleophiles. The fact that an amine nucleophile adds to a double bond with a half-life of a few seconds at $25^{\circ} \mathrm{C}$ is nothing short of astounding. In the second reaction of Fig. 5, two hydrogens jump across space to reduce a double bond, an equally amazing process. Both the amine addition and the reduction illustrate the principle that enzyme-like rates and chemistry occur when the reactive entities lie, without the possibility of escape, within bonding distances of each other. 
<smiles>COc1ccc(/C=C/c2cc3c(cc2CCN(C)CC(C)C)OCO3)c(CN(C)C(C)C)c1OC</smiles>

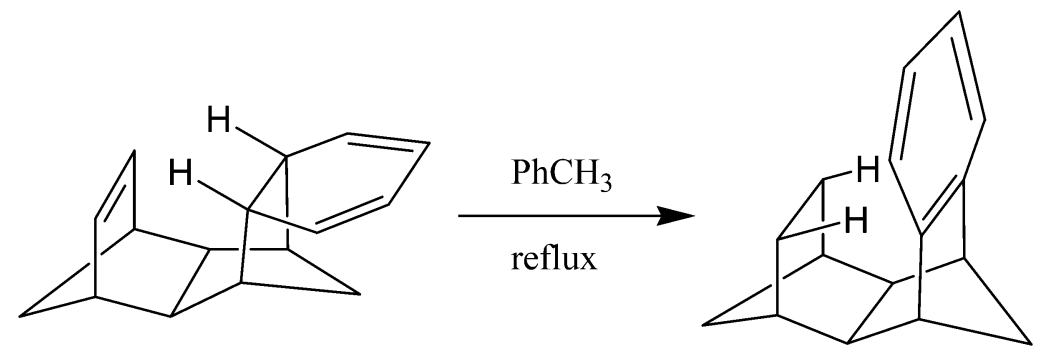

Fig. 5 Extremely fast intramolecular reactions occurring when contact distances are rigidly imposed upon the reactive groups.

Figure 6 illustrates a corollary to the above principle: Even a single free rotation that permits the reactants to depart from bonding distances can have a highly deleterious effect upon the rate. Both reactions in Fig. 6 involve a backside $S_{N} 2$ displacement to form a three-membered ring [20]. Strain considerations favor the reaction of acyclic amine over that of the bicyclic amine because a greater strain is created in the product of the bicyclic reaction. Yet, the bicyclic compound reacts five orders of magnitude faster than the acyclic compound. Why should this be? According to our corollary, the amine in the bicyclic compound cannot escape the backside of the reactive carbon, whereas in the acyclic case a free rotation allows the amine to do so. And this time element makes all the difference.

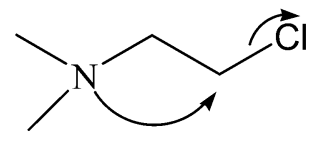

$\mathrm{k}_{\text {rel }}=1$

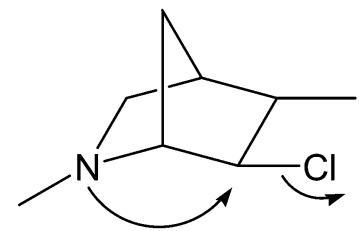

$\mathrm{k}_{\mathrm{rel}}=2 \times 10^{5}$

Fig. 6 Demonstration of how even a modest rotational freedom in the left-hand compound has a deleterious effect on the rate of $\mathrm{S}_{\mathrm{N}} 2$ displacement.

We are now in a position to express the "spatiotemporal hypothesis": Enzyme reactions are fast because the substrate is held rigidly at a contact distance from the enzyme's catalytic groups. Thus, enzymatic rates are rationalized in terms of time and distance, both of which are simple, understandable, and potentially measurable parameters. By "contact distance" is meant a distance less than the diameter of water (ca. $3 \AA$ ). When, for example, a nucleophile is forced to reside $2.5 \AA$ above a carbonyl carbon, one can expect an enzyme-like rate for a carbonyl addition.

Bruice and coworkers have advanced the notion that so-called "near-attack conformations" (NACs) play a key role in enzyme catalysis [21]. This theory basically reiterates the tenets of the earlier spatiotemporal hypothesis. Focusing as they do on ground states, neither spatiotemporal nor NAC ideas invoke transition-state properties. The main difference is that NAC incorporates a reactive "space" 
as opposed to the emphasis on distance embodied in spatiotemporality. Although it is much more difficult to define a volume than a distance, the NAC concept is a valuable, although a trifle redundant, contribution to enzyme catalysis.

But, along similar lines, exactly how original is the spatiotemporal theory? Is it not merely a thinly disguised rehashing of familiar concepts including entropy, proximity, and intramolecularity? Obviously spatiotemporal effects embody these three concepts. Imposing time and distance constraints on two reactants necessarily brings them into proximity with attendant entropic consequences. That is why I have been careful not to call other enzyme theories "wrong". The truth, it is important to understand, is not under discussion here. The problem in enzyme catalysis is not to voice the truth but to formulate a model, and models are to be used, not believed. It is our contention that entropy, etc. are not very useful models, whereas spatiotemporality, relying as it does on definable time and distance parameters, is potentially more predictive and thus more informative.

Years ago I derived an equation relating rate and distance [4,10]. It is based on two overlapping parabolas that are shifted together horizontally (as opposed to vertically as in Marcus theory), thereby lowering the transition state (corresponding to the point where the parabolas intersect). The equation (eq. 2) shows that the activation energy $E_{\mathrm{a}}$ is proportional to $d^{2}$, the distance squared

$$
E_{\mathrm{a}}=\mathrm{F} d^{2} / 8
$$

between the reacting atoms. Since rate constants are logarithmically related to activation energy, rate constants are extraordinarily sensitive to distance when distances fall below $3 \AA$. A few tenths of an angstrom can lead to orders-of-magnitude differences in rate. Admittedly, the construct is approximate since it assumes, for example, that the parabolas do not change their shape as they approach one another. Yet the conclusion is obvious: Simple theoretical ideas support a close relationship between rate and distance.

Naturally, we sought experimental verification of the rate/distance equation given in eq. 2 . This turned out to be more difficult than might be at first guessed. Ideally, one would need a set of molecules whose distance between intramolecular reactive atoms can be varied, say, a tenth of an angstrom at a time. Moreover, reaction between the reactive atoms should not introduce extraneous variables, such as strain, within the set. Rather accidentally, we finally encountered a system that did allow us to estimate a rate/distance dependence. The basis of this study goes back to 1956 when Bunnett and Okamoto reported that the Smiles rearrangement in Fig. 7 experiences a remarkable $0.5 \times 10^{6}$ rate increase when $\mathrm{R}$ is changed from $\mathrm{H}$ to $\mathrm{Me}$ [22]. We performed ab initio calculations on these two compounds plus analogs in which $\mathrm{R}=i$-Pr and $t$-Bu. The results are shown in Table 3 . It turns out that the $E_{\mathrm{a}}$ diminishes from $6.6 \mathrm{kcal} / \mathrm{mol}$ to $0.2 \mathrm{kcal} / \mathrm{mol}$ as the "attack distance" (i.e., the distance between oxygen anion and the electrophilic carbon) diminishes from 3.05 to $2.58 \AA$. For $\mathrm{R}=\mathrm{H}, \mathrm{Me}$, and $i$-Pr, there is indeed a linear relationship between $E_{\mathrm{a}}$ and $d^{2}$. For $\mathrm{R}=t$ - $\mathrm{Bu}$, the reaction is too fast to measure accurately. In summary, a decrease from 3.05 to $2.80 \AA$ leads to a rate increase of 3.6 powers of 10 in the gas phase and almost six powers of 10 in solution. Ground-state distance is critical to organic reactivity and, by inference, to enzymatic reactivity.
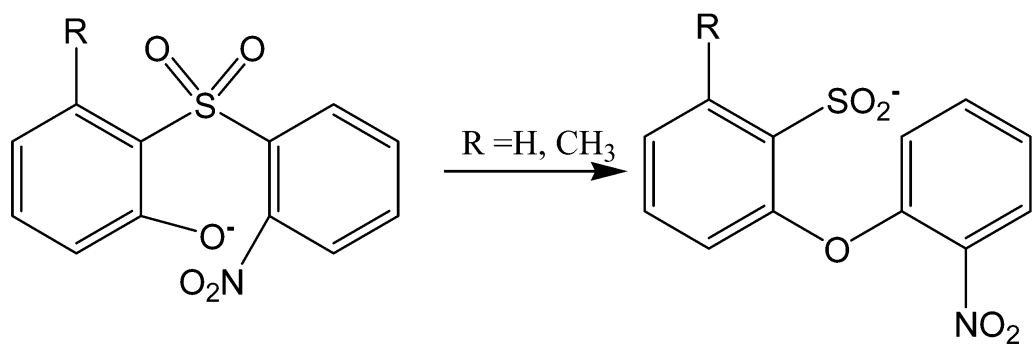

Fig. 7 A Smiles rearrangement which was the focus of ab initio calculations relating rate to distance. 
Table 3 Geometric and energy data for four Smiles rearrangements.

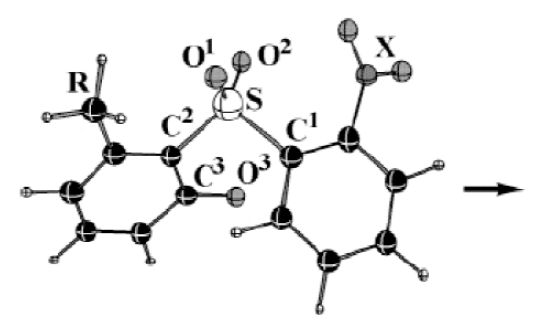

1

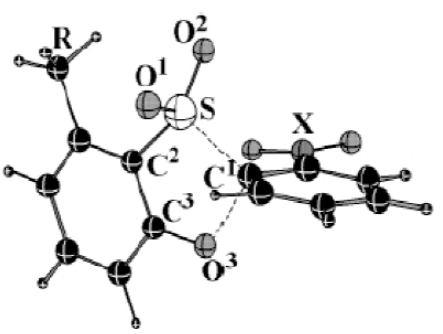

2

Transition State (TS)

\begin{tabular}{lcccc}
\hline $\mathrm{R}$ & $\mathrm{O}^{3}-\mathrm{C}^{1} ;(\mathrm{GS}) / \AA$ & $\mathrm{O}^{3}-\mathrm{C}^{1} ;(\mathrm{TS}) / \AA$ & $\mathrm{O}^{3}-\mathrm{C}^{1}-\mathrm{S} /{ }^{\circ}$ & $E_{\mathrm{a}} / \mathrm{kcal} \mathrm{mol}^{-1}$ \\
\hline $\mathrm{H}$ & 3.05 & 1.74 & 72.4 & 6.6 \\
$\mathrm{Me}$ & 2.80 & 1.77 & 74.3 & 1.5 \\
$i-\mathrm{Pr}$ & 2.67 & 1.79 & 74.9 & 0.3 \\
$t-\mathrm{Bu}$ & 2.58 & 1.80 & 75.5 & 0.2 \\
\hline
\end{tabular}

B3LYP/6-31G(d,p) and B3LYP/6-311+G(d,p) levels. IBM SP-Power 3 (375 MHz) supercomputer.

If spatiotemporal theory has any merit, then it should be possible to design a priori a system in which short, imposed distances permit an otherwise negligible reaction. This was accomplished in a study of amide hydrolysis (a normally recalcitrant reaction requiring reflux in strong acid for many hours) using the amide shown in Fig. 8 [3]. Owing to the 1,3-diaxial relationship between carboxyl and amide, the amide hydrolyzes in minutes at room temperature and neutral $\mathrm{pH}$. This rate, equaling or exceeding that of a protease-catalyzed hydrolysis, can be ascribed to the short ( $2.8 \AA$ ) distance between carboxyl oxygen and amide carbonyl carbon prior to formation of an anhydride intermediate. Simultaneous proton transfer is also likely. Now, one might claim that the remarkably fast amide cleavage stems from "steric compression". But, we can evaluate the magnitude of this steric compression because it must be less than the energy difference between the favored triaxial comformation shown in Fig. 8 and the corresponding triequatorial conformation. Were steric compression to exceed this energy difference, the molecule would simply flip into the triequatorial conformation. It turns out that the estimated maximum possible steric compression accounts for only a small fraction of the huge rate acceleration. All an enzyme need do, therefore, is align one of its carboxyls, relative to the substrate's amide carbonyl, in the geometry shown in Fig. 8, and the typical enzyme catalysis will be achieved. We have obtained this alignment via covalent bonds, but no one has yet modeled the alignment with an intermolecular chemical system. Biomimetic chemistry remains decidedly inferior to enzymology. 


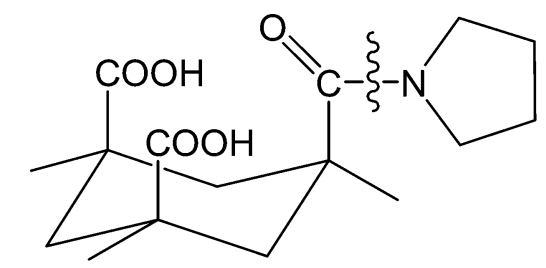

$$
\mathrm{t}_{1 / 2}=8 \min @ \mathrm{pD}=7.05,21.5^{\circ} \mathrm{C}
$$

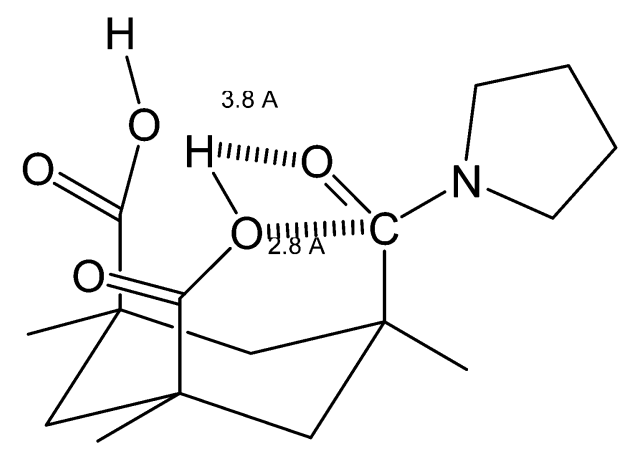

Fig. 8 An amide that hydrolyzes in minutes at neutral $\mathrm{pH}$ and room temperature owing to spatiotemporal effects.

\section{SPLIT-SITE MODEL OF ENZYMES}

The remainder of this paper will focus on the enzymes themselves. In order to interpret enzyme behavior, we have found it necessary to invoke what we call the "split-site" model [7]. As everyone knows, both substrates and enzymes have a binding site and a reactive site as portrayed in Fig. 9. According to the figure, binding of the substrate at its binding site $\mathrm{B}$ forces its reactive group $\mathrm{R}$ into a contact distance with catalytic groups on the enzymes (X and $\mathrm{Y}$ ). We have already discussed at length what this rigidly imposed ground-state distance, shorter than the diameter of water, has on the reaction rate.
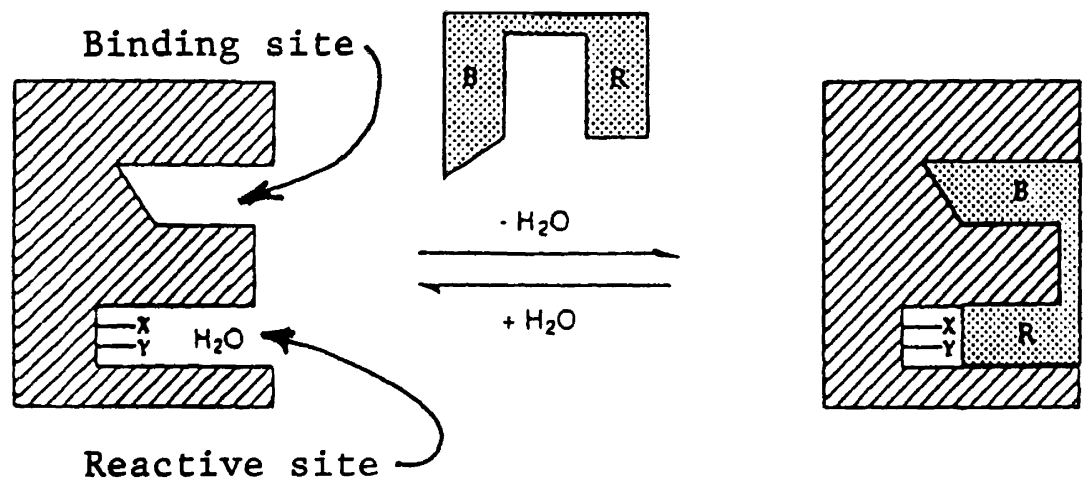

Fig. 9 The split-site model treating an enzyme's reactive site and the binding site as separate entities. 
Although few will argue with the split-site model, analyses of enzyme energetics seldom invoke the model. This is unfortunate because when the model is ignored, then the very essence of enzyme action is lost. For a full understanding of enzymes, one needs to treat B and R separately. This is vividly demonstrated from the "fundamentalist" position expressed by Schowen in 1978 and still advanced today in the texts and literature: "Reactant state interactions are by nature inhibitory and only waste catalytic power." Let us examine, with the aid of Fig. 10, how enzymologists arrived at this conclusion.
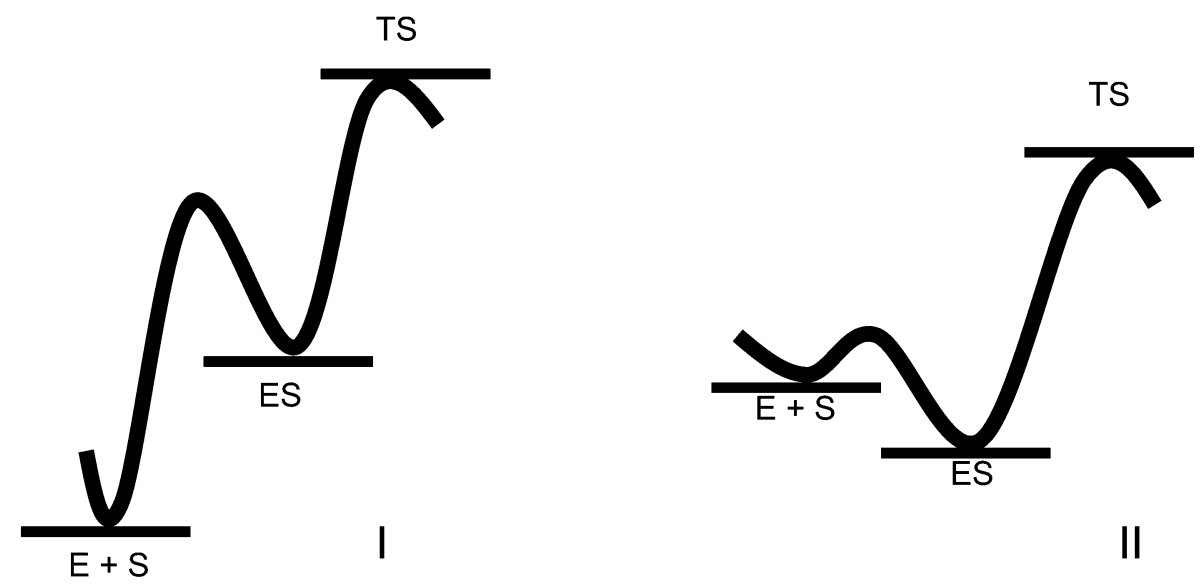

II

Fig. 10 Energy profiles of an enzyme-catalyzed reaction in which ES is of higher energy than $(E+S)$ in case I and of lower energy in case II.

If one neglects to subdivide enzyme interactions into its components, then there are only two possibilities: ES must either lie above $(E+S)$ or below $(E+S)$. If $E S$ lies above $(E+S)$, then lowering or raising the ES energy has no effect on the rate because the rate depends only upon the energy difference between transition state TS and $(E+S)$. In other words, the exact $(E+S)$ to TS pathway is irrelevant to the rate. If, on the other hand, ES lies below $(E+S)$, then lowering the ES energy will decrease the rate by increasing the energy difference between ES and TS. Hence, one can conclude that ES either does nothing for the rate (case I) or actually inhibits the rate (case II).

Why, one may ask, does the enzyme-substrate ES form at all if it has either no effect, or a deleterious effect, upon the rate? "For the sake of stereochemistry," one might conjecture. But this cannot be true because simple bimolecular reactions, such as the Diels-Alder and $\mathrm{S}_{\mathrm{N}} 2$ reactions, are stereospecific, yet they do not (usually) form an intermediary complex. Stereochemistry is not predicated on complex formation. We are thus left with a dilemma. All known enzymes without exception form an ES complex, yet we do not know why.

Now, let us analyze the situation when the ES complex is subdivided into a binding site and a reaction site. To begin with, I have assigned in Fig. 11 arbitrary energy values of 0 to $(E+S) ;+10$ to ES; and +17 to TS. It is easier to discuss a concept with concrete numbers, arbitrary though they might be, than to do so abstractly. Below the energy profile an "old" enzyme is converted into a "new" enzyme by my tinkering with the ES energy levels in the context of the split-site model. Thus, $\mathrm{ES}_{\mathrm{B}}$ and $\mathrm{ES}_{\mathrm{R}}$ are initially given -3 and +13 energy units, respectively, which are values that add up to the proscribed ES energy of $+10 . \mathrm{ES}_{\mathrm{B}}$ is negative because hydrogen-bonding, etc. at the binding site is favorable, whereas $\mathrm{ES}_{\mathrm{R}}$ at the reactive site is positive because it costs energy to desolvate reactive groups and to otherwise hold them at contact distances.

Next, it is necessary to mention an important principle in our analysis of the split-site model: Binding energy in ES is conserved in the TS. For example, in the above case, $\mathrm{ES}_{\mathrm{B}}=\mathrm{TS}_{\mathrm{B}}=-3$. This is a fair assumption because, as a first approximation, nothing happens at the binding site as ES transforms 


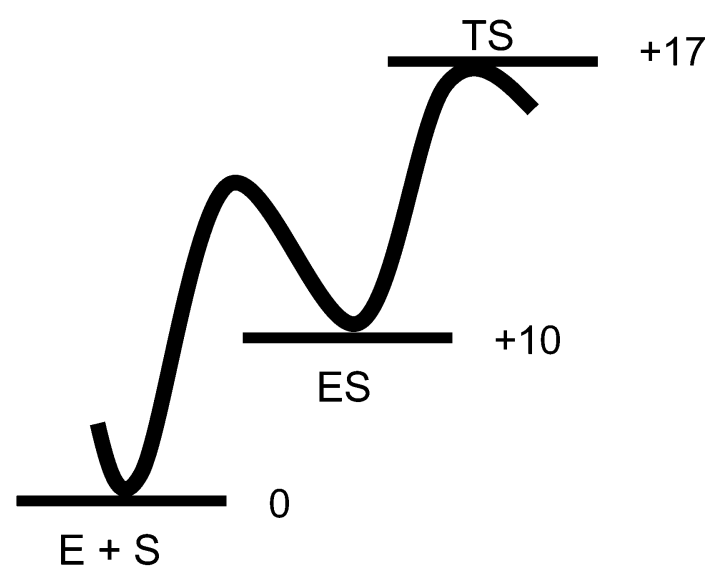

\begin{tabular}{|l|r|r|}
\hline & \multicolumn{1}{l|}{ Old } & \multicolumn{1}{l|}{ New } \\
\hline $\mathrm{E}+\mathrm{S}$ & 0 & 0 \\
\hline $\mathrm{ES}_{\mathrm{B}}$ & -3 & -4 \\
\hline $\mathrm{ES}_{\mathrm{R}}$ & +13 & +13 \\
\hline $\mathrm{ES}$ & +10 & +9 \\
\hline $\mathrm{TS}_{\mathrm{B}}$ & -3 & -4 \\
\hline $\mathrm{TS}_{\mathrm{R}}$ & +20 & +20 \\
\hline $\mathrm{TS}$ & +17 & +16 \\
\hline$\Delta \mathrm{G}^{\ddagger}$ & +17 & +16 \\
\hline effect & & accel \\
\hline
\end{tabular}

Fig. 11 Case 1. ES lies above $E+S\left([S]<K_{m}\right)$. Creating a new and faster enzyme by stabilizing the binding at the binding site.

into TS. Since $\mathrm{TS}_{\mathrm{B}}=-3$, and since $\mathrm{TS}=+17$, it follows that $\mathrm{TS}_{\mathrm{R}}=+20$. The activation free energy of the reaction, $\mathrm{TS}-(\mathrm{E}+\mathrm{S})$, thus equals +17 .

Now let us create a new enzyme by strengthening the binding of $\mathrm{ES}$ (i.e., changing $\mathrm{ES}_{\mathrm{B}}$ from -3 to -4) but leaving $\mathrm{ES}_{\mathrm{R}}$ alone. This could happen, for example, by adding a hydrogen bond between the enzyme and substrate somewhere distant from the site where the actual chemistry takes place. It can be seen from Fig. 10 that this modification of ES lowers TS from +17 to +16 because binding is conserved. In other words, the reaction is accelerated with the new enzyme.

It is true that the reaction is accelerated by lowering TS. But, as shown above, this acceleration can derive from an additional conserved interaction in the ground state. An analysis that ignores the split-site properties of enzyme reactions, and handles ES as a single energy term, would incorrectly deduce that adding a hydrogen bond distant from the reactive site might stabilize ES, but it would not necessarily affect the enzyme rate at the reaction site.

A similar line of thinking was applied to the case where ES lies below $(E+S)$ in Fig. 12. Here, the ES level of the old and new enzymes was kept constant at -4 . In so doing, there can be no concern that the ES concentration changes as the new enzyme if formed. But the -4 is composed of $\mathrm{ES}_{\mathrm{B}}=-7$ and $\mathrm{ES}_{\mathrm{R}}=+3$ in the old enzyme, in contrast to $\mathrm{ES}_{\mathrm{B}}=-8$ and $\mathrm{ES}_{\mathrm{R}}=+4$ in the new enzyme. As seen, this leads to an overall rate acceleration. Mechanistically, and in accord with spatiotemporality, this means that a tighter binding (more negative $\mathrm{ES}_{\mathrm{B}}$ ) that forces the reactive groups closer together (more positive $\mathrm{ES}_{\mathrm{R}}$ ) accelerates the rate even though the energy level of ES remains constant. This is a totally different statement from the fundamentalist position that ES is either irrelevant or deleterious to the rate. 


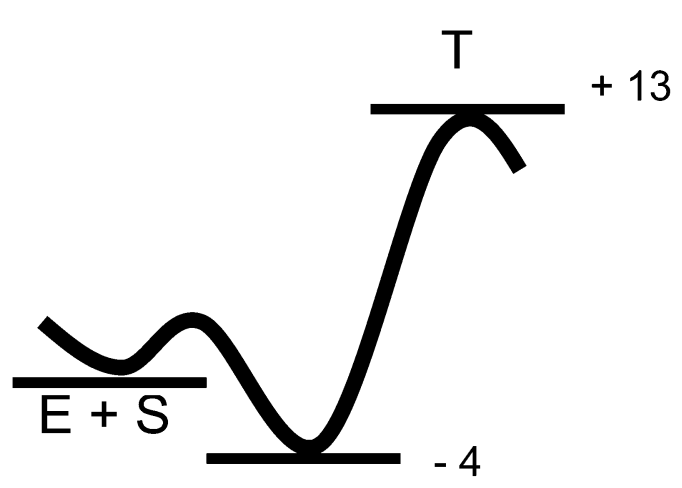

\begin{tabular}{|l|r|r|}
\hline & \multicolumn{1}{l|}{ Old } & \multicolumn{1}{l|}{ New } \\
\hline $\mathrm{E}+\mathrm{S}$ & 0 & 0 \\
\hline $\mathrm{ES}_{\mathrm{B}}$ & -7 & -8 \\
\hline $\mathrm{ES}_{\mathrm{R}}$ & +3 & +4 \\
\hline $\mathrm{ES}$ & -4 & -4 \\
\hline $\mathrm{TS}_{\mathrm{B}}$ & -7 & -8 \\
\hline $\mathrm{TS}_{\mathrm{R}}$ & +20 & +20 \\
\hline $\mathrm{TS}$ & +13 & +12 \\
\hline$\Delta \mathrm{G}^{\ddagger}$ & +17 & +16 \\
\hline effect & & accel \\
\hline
\end{tabular}

Fig. 12 Case 2. ES lies below $E+S\left([S]>K_{m}\right)$. Creating a new and faster enzyme by stabilizing the binding at the binding site while elevating the energy at the reactive site.

In conclusion, I reproduce a schematic of an active site taken from Kraut et al.'s recent review [23] on enzyme catalysis (Fig. 13). The figure portrays an enolase whose carboxylate removes a proton from a ketone substrate. Although not stated as such, the drawing embodies the spirit of spatiotemporal theory. Thus, multiple noncovalent binding imposes a contact distance upon a carboxylate oxygen and the labile proton. Not even a water molecule separates the two reactive atoms. It is this close contact that allows the proton to "swim upstream" by perhaps $15-20 \mathrm{pKa}$ units (i.e., a very weak base removes a proton from a very weak acid). Of course, transition-state stabilization, arising from hydrogen-bonding effects as the ketone carbonyl assumes a greater negative charge, plays a role in the catalysis as well. Although spatiotemporal effects in the ground state may not be the exclusive source of catalysis, I hope I have made a cogent case that they are a component of enzymology to be reckoned with.

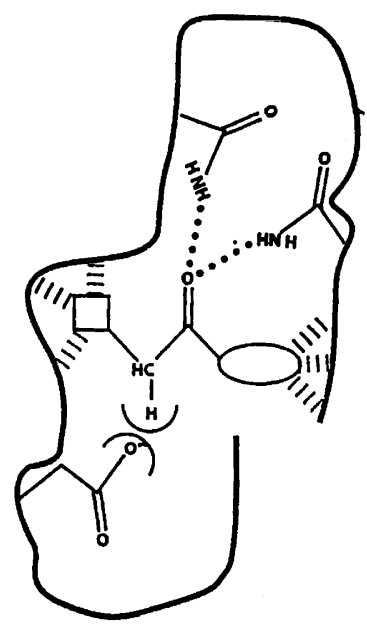

Fig. 13 A schematic of an enolase enzyme incorporating the tenets of spatiotemporal theory. Reproduced with permission from ref. [23]. 


\section{ACKNOWLEDGMENTS}

This work was supported by the National Institutes of Health. The author thanks the many wonderful students who have passed through his laboratory and who have done all the work.

\section{REFERENCES}

1. F. M. Menger. Acc. Chem. Res. 18, 128 (1985).

2. F. M. Menger and U. V. Venkataram. J. Am. Chem. Soc. 107, 4706 (1985).

3. F. M. Menger and M. Ladika. J. Am. Chem. Soc. 110, 6794 (1988).

4. F. M. Menger. Adv. Mol. Modeling 1, 189 (1988).

5. M. J. Sherrod and F. M. Menger. Tetrahedron Lett. 31, 459 (1990).

6. F. M. Menger and K. Gabrielson. J. Am. Chem. Soc. 114, 3574 (1992).

7. F. M. Menger. Biochemistry, 31, 5368 (1992).

8. F. M. Menger. Acc. Chem. Res. 26, 206 (1993).

9. N. A. Khanjin, J. P. Snyder, F. M. Menger. J. Am. Chem. Soc. 121, 11831 (1999).

10. F. M. Menger, A. L. Galloway, D. G. Musaev. Chem. Comm. 2370 (2003).

11. L. Pauling. Nature 161, 707 (1948).

12. K. N. Houk, A. G. Leach, S. P. Kim, X. Zhang. Angew. Chem., Int. Ed. 42, 4872 (2003).

13. R. H. Abeles, P. S. Frey, W. P. Jencks. Biochemistry, Jones and Bartlett, Boston (1992).

14. R. Bird and C. J. M. Stirling. J. Chem. Soc., Perkin Trans. 21221 (1973).

15. D. F. DeTar and N. P. Luthra. J. Am. Chem. Soc. 102, 4504 (1980).

16. D. R. Storm and D. E. Koshland. J. Am. Chem. Soc. 94, 5805 (1972).

17. F. M. Menger and L. E. Glass. J. Am. Chem. Soc. 102, 5404 (1980).

18. T. C. Bruice. Annu. Rev. Biochem. 45, 331 (1976).

19. A. J. Kirby and C. J. Logan. J. Chem. Soc., Perkin Trans. 2642 (1978).

20. R. O. Hutchins and L. Rua. J. Org. Chem. 49, 1811 (1975).

21. S. Hur and T. C. Bruice. J. Am. Chem. Soc. 125, 10540 (2003).

22. J. F. Bunnett and T. Okamoto. J. Am. Chem. Soc. 78, 5363 (1956).

23. D. A. Kraut, K. S. Carroll, D. Herschlag. Annu. Rev. Biochem. 72, 517 (2003). 